\title{
Sex differences in the use of social information emerge under conditions of risk
}

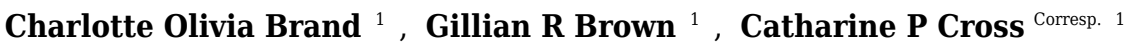 \\ ${ }^{1}$ School of Psychology and Neuroscience, University of St. Andrews, St Andrews, United Kingdom \\ Corresponding Author: Catharine P Cross \\ Email address: cpc2@st-andrews.ac.uk
}

Social learning provides an effective route to gaining up-to-date information, particularly when information is costly to obtain asocially. Theoretical work predicts that the willingness to switch between using asocial and social sources of information will vary between individuals according to their risk tolerance. We tested the prediction that, where there are sex differences in risk tolerance, altering the variance of the payoffs of using asocial and social information differentially influences the probability of social information use by sex. In a computer-based task that involved building a virtual spaceship, men and women ( $\mathrm{N}=88$ ) were given the option of using either asocial or social sources of information to improve their performance. When the asocial option was risky (i.e., the participant's score could markedly increase or decrease) and the social option was safe (i.e., their score could slightly increase or remain the same), women, but not men, were more likely to use the social option than the asocial option. In all other conditions, both women and men preferentially used the asocial option to a similar degree. We therefore found both a sex difference in risk aversion and a sex difference in the preference for social information when relying on asocial information was risky, consistent with the hypothesis that levels of risk-aversion influence the use of social information. 
6 Word count $=4,223$, excluding references; abstract $=214$

$7 \quad$ * Corresponding author:

8 Dr Catharine Cross,

9 School of Psychology \& Neuroscience,

10 University of St Andrews,

11 South Street,

12 St Andrews,

13 KY16 9JP, U.K.

14 Phone: +44 1334467234

15 Email: cpc2@st-andrews.ac.uk 


\section{Abstract}

17 Social learning provides an effective route to gaining up-to-date information, particularly when

18 information is costly to obtain asocially. Theoretical work predicts that the willingness to switch

19 between using asocial and social sources of information will vary between individuals according

20 to their risk tolerance. We tested the prediction that, where there are sex differences in risk

21 tolerance, altering the variance of the payoffs of using asocial and social information differentially

22 influences the probability of social information use by sex. In a computer-based task that

23 involved building a virtual spaceship, men and women $(\mathrm{N}=88)$ were given the option of using

24 either asocial or social sources of information to improve their performance. When the asocial

25 option was risky (i.e., the participant's score could markedly increase or decrease) and the social

26 option was safe (i.e., their score could slightly increase or remain the same), women, but not

27 men, were more likely to use the social option than the asocial option. In all other conditions,

28 both women and men preferentially used the asocial option to a similar degree. We therefore

29 found both a sex difference in risk aversion and a sex difference in the preference for social

30 information when relying on asocial information was risky, consistent with the hypothesis that

31 levels of risk-aversion influence the use of social information. 


\section{Introduction}

33 Individuals can acquire information either directly through their own asocial learning experiences

34 or by copying other individuals [1]. Asocial learning allows individuals to gain first-hand

35 knowledge about the immediate environment, but reliance on this type of learning can be costly,

36 for instance, in terms of time and energy [2]. In contrast, social learning can provide a cost-

37 effective route to gaining up-to-date information, particularly when the environment is changing

38 and information is costly to obtain asocially [2, 3]. Theoretical models support the hypothesis that

39 an increased reliance on social learning is adaptive when the environment becomes more

40 variable (although not when variability is very high) and when the returns from asocial learning

41 become more unreliable [3-6]. Therefore, individuals are predicted to be sensitive to the

42 reliability of the available sources of information and to use these reliability estimates when

43 choosing whether to learn asocially or socially [2].

Reliability can include the predictability of the source of information (e.g., the likelihood that a food reward is associated with a particular cue) and the variability in the expected payoff derived from different sources (e.g., the variability in the amounts of food obtained from different

47 foraging patches). Empirical research on non-human animals and humans has shown that

48 individuals are likely to use social learning when personal experience reveals that the

49 environment is unpredictable or the variability in payoffs of available options is high (e.g., [7-10]).

50 For example, a study of nine-spined sticklebacks (Pungitius pungitius) showed that reducing the

51 predictability of personally-experienced cues in a foraging context increased reliance on social

52 learning [10]. Similarly, when faced with the option of a risky or safe action in an experimental

53 paradigm, human participants were found to delay their decision and observe the choices made

54 by others, especially if their private information did not support the risky action [7]. These

55 findings support the broader hypothesis that social learning is used strategically [11]. 
58 sources sooner than risk-prone individuals when faced with unreliable personal experience. In

59 real-world scenarios, the predictability and riskiness of sources of information are likely to co-

60 vary; for example, food items with high nutritional value are likely to be both rarer in the

61 environment, and more difficult to obtain, than low value food items [4, 13]. By switching to social

62 sources of information when faced with risky options, individuals are thus potentially better able

63 to exploit high-value resources. In both non-human animals and humans, individuals vary in their

64 sensitivity to experiencing gains and losses [14, 15], and a small number of studies of non-

65 human animals have revealed that 'shy' individuals are more likely than 'bold' individuals to copy

66 the behaviour of others (e.g., [16-19]). However, the link between risk-proneness and social

67 information use has yet to be evaluated in humans.

68 One variable that is commonly related to risk aversion in humans is an individual's sex,

69 with women obtaining lower average scores than men on a range of risk-taking measures (e.g.,

70 [20-22]). While the degree of overlap between the sexes on risk-aversion measures is often

71 considerable [23], and not all risk-aversion measures show sex differences [24], women

72 perceive the benefits gained from taking risks as being lower than do men [24, 25]. Women also

73 rate both the likelihood of a negative outcome and the perceived severity of the costs higher

74 than do men [24, 25], and report being less likely than men to engage in novel activities that

75 involve risk [22]. Similarly, data from personality measures indicate that, on average, women are

76 more sensitive than men to the potential negative outcomes of decisions [26]. The probability of

77 using asocial versus social sources of information when faced with a risky decision is therefore

78 likely to differ on average between women and men.

The aim of this study was to examine whether altering the riskiness of using asocial and social sources of information would differentially influence the probability that men and women

81 used these sources. Here, we are defining riskiness in terms of variation in expected score [27].

82 We predicted that, when one of these sources of information appeared to be risky (i.e., high

83 variation in expected score), women would be more likely than men to use the alternative 
84 source, safe (i.e., low variation in expected score) of information. In the control condition, no sex

85 difference in the use of asocial and social sources was predicted. We designed a novel

86 computer-based task that involved constructing a virtual spaceship. After building the first

87 spaceship, participants were given the option of using asocial or social sources of information to

88 improve their ranked score. Participants were assigned to one of three conditions, in which

89 either i) the asocial option was risky and the social option was safe, ii) the asocial option was

90 safe and the social option was risky, or iii) both the asocial and social options were safe.

91 Because scores were randomly allocated to spaceships, participants could not learn about the

92 usefulness of different design features. The outcome measure of principal interest was the

93 participant's choice of information source. Participants also completed a risky impulsivity

94 measure [28], in order to confirm that the predicted sex difference in average score was found in

95 our set of participants.

\section{Methods}

97 Participants

98 Eighty-eight participants (50 women and 38 men) were recruited through the University of St

99 Andrews' School of Psychology \& Neuroscience online participant recruitment system. All

100 participants were aged 17 or over, with the majority (91\%) falling into the $18-25$ age range.

101 Participants gave consent before taking part in the experiment and were debriefed afterwards.

102 All participants were reimbursed $£ 3$ for attending the session - which lasted approximately 20

103 minutes - and could obtain an additional $£ 2$ depending on performance criteria (see 'Procedure'

104 below). Participants were randomly assigned to one of the three conditions (see 'Asocial and

105 social information') and were tested in groups (range $=4-9$ individuals). Participants gave

106 consent via a button click at the start of the experiment. This was approved, as were all other

107 procedures used in this study, by the Ethics Committee of the School of Psychology \& 
108 Neuroscience on behalf of the University of St Andrews (approval code PS11481).

109

110 Participants stated their gender ('female', 'male'), age bracket, current level of education and

111 country of origin before beginning the experiment. They then played a computer game,

112 programmed using web-based JavaScript, in which they built virtual spaceships. Participants

113 were instructed that the aim was to construct spaceships with the highest scores, and that the

\section{Procedure}

\begin{abstract}
participant with the highest score at the end of the session would receive a bonus payment.
\end{abstract}
Spaceship construction proceeded in three rounds, each with two building phases. In Phase 1, participants constructed their first spaceship by selecting tiles from a grid of thirty available items that were arranged into themes (crew, cargo, engines, shields and lasers) (see Figure 1).

Players had two minutes to view these items and choose ten to place on a spaceship template.

The only constraint was that they had to use at least one crew member and one engine. After finishing Phase 1, each player's ship was given a numerical score and a rank in a league table (1st to 5th, highest to lowest) (see Figure 2). Players were given no information on how a good score might be achieved, and, in reality, scores were randomly assigned to the participants' spaceship (with a range of 8,000 to 25,000 ), along with a false 'rank' that was always either 1 st, 3rd or 5th.

Participants then chose between using asocial and social sources of information (see 'Asocial and social options') (see Figure 3) before building a second spaceship (Phase 2). Participants were not given a score or rank for their second spaceship at the end of Phase 2, meaning that they received no feedback on whether the choice to use asocial or social source of information improved the outcome. Furthermore, because scores were randomly generated, no rules for building high-scoring spaceships were available for participants to learn.

Phases 1 and 2 were then repeated a further two times (i.e., three Rounds of building spaceships), with scores and ranks shown at the end of each Phase 1. Each participants' 
133 spaceships were ranked randomly, once at $1 \mathrm{st}$, $3 \mathrm{rd}$ or $5^{\text {th }}$. These rankings were displayed on a

134 league table to the participants. At the end of each Round, participants were informed that their

135 score had been saved and that their best score out of the three Rounds would be used at the

136 end of the experiment to allocate the bonus. Because the scores given to spaceships were

137 random, bonuses were awarded at the end of the experiment to more than one participant

138 according to a lottery in which participants had a higher probability of a reward when choosing

139 the safe rather than the risky option in the final Round.

140 After completing all three Rounds, participants completed the 12-item risky impulsivity

141 measure [28] on-screen. This measure assesses willingness to take risks without prior thought in

142 everyday life and is reported to have high internal consistency [28]. The bonus payments were

143 awarded when all participants had completed the final on-screen material, including the

144 questionnaire.

\section{Asocial and social options}

146 The asocial option consisted of viewing up to ten previously unseen items in the scrapheap, of

147 which up to three items could be kept for use in the next building Phase. The social option

148 consisted of viewing three completed spaceships, ostensibly built by 'other participants', along

149 with the associated 'scores'. These spaceship designs had actually been generated by the

150 experimenter prior to the study using randomly selected tiles, and three out of twelve completed

151 spaceships were presented at random as social sources. The scores for these spaceships were

152 also randomly assigned. Participants could choose up to three items from one of the three ships,

153 and these items were automatically added to the participant's spaceship template in the next

154 Phase and could not be removed. Because each participant was assigned to a single condition,

155 the description of the asocial and social options (see below) remained the same for participants

156 across Rounds, in order to avoid potential confusion among participants and reduce the chance

157 that participants failed to attend to the subtle differences in the descriptive material. 
158 In the Asocial Risky (AR) condition ( $\mathrm{N}=28 ; 18$ female, 10 male), participants were informed

159 that their score could markedly increase or decrease if they visited the scrapheap, in a short

160 paragraph that included the following wording: "some of these items may be broken and useless,

161 but some may greatly increase your ship's score... your score could go up or down”. Conversely,

162 the social option was safe; participants were informed their score could slightly increase or

163 would remain the same ("the ships will have the same score as your ship, or slightly higher...

164 you will be guaranteed at least the same score as your current ship"). In the Social Risky (SR)

165 condition ( $\mathrm{N}=32 ; 17$ female, 15 male), the asocial option was safe ("all of these items will help

166 your ship to fly, and some of them can slightly increase your ship's score... you will be

167 guaranteed at least the same score as your current ship"), and the social option was risky ("the

168 ships may have a much worse or much better score that your current ship's score... your score

169 could go up or down"). In the Control (C) condition ( $N=28 ; 15$ female, 13 male), both the asocial

170 and social options were safe: the wording was identical to that used in the safe options in the

171 other conditions. This wording reflects the Bounded Risk Distribution model, in which individuals

172 are expected to maximize their probability of reaching a goal while minimizing their probability of

173 falling below a certain threshold [27]. In our experiment, participants are trying to achieve the

174 goal of a top score and want to minimize their chance of falling below this threshold, in order to

175 achieve a bonus payment. Therefore, although the safe options have a slightly higher average

176 expected score, these safe options preclude a large increase in score. The risky option is

177 therefore a rational choice where participants believe that they need to greatly improve their

178 score in order to move up in the rankings and win a monetary bonus.

179 Statistical analyses

180 We modelled the participants' decision to use asocial or social options using Bayesian binomial

181 multi-level logistic regression in $\mathrm{R}$ with the map2stan function from the Rethinking package [29].

182 The full model included an effect for sex, an effect for condition, a sex ${ }^{*}$ condition interaction, an

183 effect for the rank given to the participant's spaceship after Phase 1, and a random effect for 
184 individual. The $\mathrm{C}$ condition was represented as the baseline in the model, so that any effects of

185 the AR or SR conditions were in relation to C. Because men were coded as 0 and women as 1 ,

186 the baseline represents men's behaviour in the control condition, and the effect of sex

187 represents how women's behaviour differed from men's in the C condition. Model predictions

188 were calculated by averaging across all candidate models weighted according to the WAIC

189 (Watanabe-Akaike Information Criteria). The model with the lowest WAIC value, and the highest

190 Akaike weight, is the model that is most likely to make accurate predictions on new data,

191 conditional on the set of models considered. Posterior predictions were calculated based on the

192 population mean of the participants and thus represent predictions for a 'new', previously

193 unobserved, average participant. These predictions are presented in Figure 4. Candidate

194 models were chosen based on a priori hypotheses formulated before data collection (Table 1).

195 In order to examine whether the choice of using risky or safe options varied with the rank

196 assigned to the spaceship, or sex of participant, and whether men and women responded

197 differently to their rank assignments, we ran an additional model with risky/safe choice rather

198 than social/asocial choice as the outcome variable. This model excluded data from the C

199 condition, because both options in this condition were safe and therefore no risky choice could

200 be made. The risky/safe choice was modelled using a Bayesian binomial multi-level logistic

201 regression with rank, sex and a sexrank interaction as predictors.

202 Finally, we also modelled participants' risky impulsivity scores using a Bayesian linear

203 model, with sex as a predictor variable, to check whether our sample displayed the expected sex

204 difference in risky impulsivity.

205 All model estimates are reported with $89 \%$ credible intervals (Cls), which are the default in

206 the Rethinking package [28]. The Cls provide an upper and lower estimate around the mean of

207 the parameter estimate and encompass $89 \%$ of the posterior distribution. This method contrasts

208 with the traditional use of $95 \%$ confidence intervals in null hypothesis testing. Using $95 \%$

209 intervals would not change the interpretation of our results, because we are using a model 
210 comparison approach, and the size of the credible intervals does not affect which models best fit

211 the data. All error bars are $89 \%$ credible intervals and can be interpreted as the region within

212 which the model expects to find $89 \%$ of responses, given the data and the assumptions in the

213 model.

\section{Results}

215 Asocial versus social options model

216 When modelling the probability of choosing asocial or social options, the best-fitting model (i.e.,

217 the model with the lowest WAIC value) included an effect for sex and an effect for the sex and

218 AR condition interaction (Table 1). This interaction can be seen in detail in Figure 4. In the C

219 condition, both women and men preferentially chose to use the asocial source information rather

220 than the social source (women: $\beta=-0.72, \mathrm{Cl}[-1.36,-0.05]$; men: $\beta=-0.41, \mathrm{Cl}[-0.72,-0.10]$;

221 Figure 4). As shown by the model estimates (Figures 5 \& 6), there was no strong evidence for

222 an interaction effect between sex and $\operatorname{SR}$ condition $(\beta=0.62, \mathrm{Cl}[-0.09,1.40])$, meaning that

223 women's choices in the SR condition did not differ strongly from women's choices in the C

224 condition. Thus, as can be seen in the model predictions (Figure 4), both women and men

225 preferentially chose the asocial source in the SR condition also. In contrast, the interaction

226 between sex and the AR condition had a strong effect in the model $(\beta=1.76, \mathrm{Cl}[1.03,2.51]$;

227 Figure 5). As can be seen in Figure 5, women in the AR condition preferentially chose the social

228 option, whereas men's choice did not differ compared to men's choices in the control condition.

229 Thus, women in the AR condition chose the social source of information more than women in the

230 C condition, while men in the AR condition did not differ from men in the $\mathrm{C}$ condition with regard

231 to their choice. According to the full model (Figure 6), rank did not predict the choice to use

232 asocial or social options $(\beta=0.05, \mathrm{Cl}[-0.22,0.29])$. 
234 The risky versus safe model indicated that participants of both sexes preferred to use the safe

235 option overall $(\beta=-2.38, \mathrm{Cl}[-4.20,-0.56]$; Figure 7). The intercept estimates the preferences of

236 men in both conditions, showing that they had an overall preference for the safe choice, and the

237 effect of sex included zero $(\beta=1.12, \mathrm{Cl}[-0.71,3.13])$, indicating that women did not choose

238 differently from men. However, the effect of rank $(\beta=1.14, \mathrm{Cl}[0.22,1.95])$ shows that both men

239 and women were more likely to choose risky than safe options after receiving a lower rank than

240 a higher rank. There was no evidence for an interaction between rank and sex in the model $(\beta=$

$241-0.77, \mathrm{Cl}[-1.98,0.19])$, indicating that men and women were responding similarly to their rank

242 assignments.

243 Risky impulsivity measure

244 Women scored lower than men on the risky impulsivity measure, as expected (women $=23.41 \pm$

$2456.97 ;$ men $=27.24 \pm 7.59 ;$ means and SEMs $)(\beta=-0.06, \mathrm{Cl}[-0.09,-0.04]$; Figure 8). Men scored

246 half a standard deviation higher than women on average (Cohen's $d=0.52$ ). The scale had an

247 acceptable level of internal consistency (Cronbach's alpha $=0.78$ ). Individual scores did not

248 predict the use of risky versus safe options $(\beta=0.13, \mathrm{Cl}[-1.75,1.90])$.

\section{Discussion}

250 In our experimental study, we found a sex difference in the choice to use social information that

251 only emerged when the alternative, asocial option was risky. Women, but not men, preferentially

252 chose to use the social source of information when the asocial option was risky. In contrast,

253 women and men did not differ from each other in their responses to risky social options; both

254 women and men preferentially used the asocial option in the 'Social Risky' condition, as well as

255 in the control condition. Male and female participants were more likely to choose the risky option 
256 when the spaceship was given a low rank than a high rank, while rank did not predict whether

257 participants chose asocial or social information. Women had lower average scores than men on

258 a risky impulsivity measure, as reported in previous research (e.g., [28, 30]). Our main finding,

259 which was that individuals of the more risk-averse sex (i.e. women) used the social option when

260 the asocial one was risky, is consistent with the hypothesis that levels of risk-aversion influence

261 social learning strategies [4]. This result has potentially broad implications for understanding the

262 dynamics of social information transmission.

263 While previous research has suggested that women are more likely than men to conform

264 to the decisions of others (e.g., [31]), our findings contribute further evidence that social sources

265 of information are used strategically, by both men and women. We found that the sex difference

266 in the use of social sources of information depended upon the type of decision being made.

267 Women were not more likely than men to use social options across all conditions, nor were

268 women less likely than men to choose the risky option in general. The sex difference in the use

269 of the social sources of information when the asocial source was risky could potentially have

270 reflected lower confidence in one's own performance in women compared to men. Previous

271 research has shown that both female and male participants copy others when lacking

272 confidence in their personal information (e.g. [31]), and that this relationship is likely to influence

273 patterns of conformity in cases where men's and women's confidence differs [32]. However, the

274 absence of a sex difference in the control condition suggests that both sexes were equally

275 confident in solving the task alone.

276 The psychological mechanisms underpinning the sex difference in response to risky

277 asocial sources remain to be determined. While a sex difference in competitiveness has been

278 identified in previous literature [33], this sex difference is unlikely to explain our results because

279 men and women responded similarly to their Phase 1 ranks. One possible explanation is that

280 women were more sensitive on average than men to the potential loss in score associated with

281 the risky asocial option and were thereby minimizing their probability of a loss. However, women

282 and men did not differ in their probability of selecting a risky social option, possibly because they 
283 had a preference for the asocial option irrespective of risk. Neither female nor male participants

284 avoided the social option completely, even when it was risky. Participants might have been

285 sampling the social sources in order to compare their own decisions with those of others or to

286 check for particularly high-scoring solutions. This sampling strategy might have prevented

287 participants from relying solely on the asocial option in the social risky condition, which might

288 have resulted in a ceiling effect. Altering the experimental design to make the social option more

289 appealing (in terms of perceived benefits gained from viewing social sources) might have

290 reduced overall reliance on asocial sources when this social information became risky

291 Our results showed that both men and women used asocial, rather than social, sources

292 of information when both sources were safe, consistent with previous experiments showing the

293 preferential use of asocial learning in laboratory settings (e.g., [32, 34]). While theoretical models

294 have suggested that social learning should initially be prioritised over asocial learning [35], our

295 empirical research suggests that participants prefer to try to solve tasks for themselves, before

296 relying on help from others. The asocial version of our task, which involved viewing new tiles in a

297 scrapheap, could have been more appealing than the social condition, in terms of providing

298 opportunities to innovate or for other reasons related to the characteristics of the stimuli.

299 Although the probability that men chose the social option did not vary across conditions, the

300 level of risk could potentially influence use of social sources of information by men under

301 different experimental conditions. For instance, further increasing the riskiness of the asocial

302 option could potentially result in men switching to using the social option. The idea that men and

303 women could differ in the cut-off point at which the risk is deemed sufficiently high to change

304 strategy could be investigated experimentally by varying the level of risk along a continuum.

305 Our results confirmed that participants of both sexes were more likely to choose the risky

306 option when the spaceship was given a low rank than a high rank. Previous experiments using

307 economic game protocols have also shown that participants are more likely to take a risk when

308 performing poorly in relation to other participants (e.g., [37, 41]). Given that our definition of

309 riskiness focused on the variation in expected score, rather than the absolute size of the 
310 expected score, future studies could manipulate both average scores and variance in scores of

311 different options to examine the influence of these on the strategic use of social information in

312 men and women. In the current study, the average score for the safe option was slightly higher

313 than for the risky option, given that scores in the safe option could either increase slightly or

314 remain stable, while scores in the risky option could either increase or decrease markedly. The

315 effects of manipulating level of risk and differences in average payoffs could be further

316 investigated experimentally in both human beings and non-human animals, using available

317 protocols (e.g., $[7,10,19])$.

318 Individual scores on the risky impulsivity measure did not correlate with the likelihood of

319 choosing the risky versus safe option. While this correlation was not the main focus of the study,

320 one suggestion for future research might be to examine measures of sensitivity to 'actuarial' risk,

321 although these measures are less likely to show sex differences than measures of sensitivity to

322 physical risk [20]. Indeed, we chose risky impulsivity because we were looking for a trait that

323 differs by sex. A second possibility is as follows. In our task, choosing the 'risky' option is rational

324 when a large increase in score is needed, but it brings with it the possibility of a large 'loss' in

325 score. We could consider a decrease in score when selecting the rational option as a form of

326 unrepresentative negative feedback [38, see also 26], to which women appear to be more

327 sensitive than men. We might therefore expect sensitivity to negative feedback in, for example, a

328 gambling task to correlate with a shift in strategy in our spaceship-building task. Consistent with

329 this explanation is the idea that women may be more sensitive to "social" punishment, in this

330 case by viewing their decline in a league table with others. Studies of different domains of risk or

331 punishment sensitivity would be welcome in order to explore these hypotheses further.

\section{Conclusion}

Our results indicated that individuals of the more risk-averse sex preferentially used a

334 social option when the asocial option was risky, supporting theoretical evidence that levels of

335 risk-aversion are linked to the implementation of social learning strategies [4]. Whether the 
336 psychological mechanisms underpinning the decision to use social sources of information

337 involved greater sensitivity to punishment or lower confidence in one's own performance was not

338 investigated. However, regardless of the mechanism, switching to social learning can potentially

339 provide individuals with the opportunity to avoid costly mistakes and learn from the successes of

340 others. Understanding how sex differences in risk-aversion relate to the use of social information

341 deserves further investigation in non-human animals, as well as humans, and would add to the

342 growing evidence that individual traits influence a broad range of social processes [12, 40].

343 Between-individual differences in risk-aversion are likely to influence the dynamics of social

344 learning and the spread of socially transmitted information through populations, with potential

345 broad-scale implications for the characteristics of local traditions and the evolution of cultural 346 traits.

\section{Acknowledgements}

348 The research was funded by a John Templeton Foundation grant, awarded to lead principal

349 investigators Kevin Laland (School of Biology, University of St Andrews) and Andrew Whiten

350 (School of Psychology \& Neuroscience, University of St Andrews). We are grateful to Ken Munro

351 for assistance with programming and to Kevin Laland and Mike Webster for comments on the

352 manuscript.

\section{Supplementary information}

354 The full dataset supporting this paper is available at https://risweb.st-

355 andrews.ac.uk/admin/workspace.xhtml?uid=5

\section{References}

1. Hoppitt W, Laland KN. 2013 Social Learning: An Introduction to Mechanisms, Methods, and

$358 \quad$ Models. Princeton University Press. 
2. Kendal RL, Coolen I, van Bergen Y, Laland KN. 2005 Trade-offs in the adaptive use of social and asocial learning. Adv. Stud. Behav. 35, 333-379.

3. Boyd R, Richerson PJ. 1985 Culture and the Evolutionary Process. Chicago University Press.

4. Arbilly M, Motro U, Feldman MW, Lotem A. 2011 Evolution of social learning when highexpected payoffs are associated with high risk of failure. J. R. Soc. Interface 8, 1604-1615.

5. Boyd R, Richerson PJ. 1988 An evolutionary model of social learning: the effects of spatial and temporal variation. In: Social Learning: Psychological and Biological Perspectives, Ed. TR Zentall, BG Galef. Laurence Erlbaum Assoc., Hillsdale, NJ. Pp. 29-48.

6. Feldman MW, Aoki K, Kumm J. 1996 Individual versus social learning: evolutionary analyses in a fluctuating environment. Anthropol. Sci. 104, 209-231.

7. Çelen B, Hyndman K. 2012 An experiment of social learning with endogenous timing. Rev. Econ. Design 16, 251-268.

8. Jones, P. L., Ryan, M. J., Flores, V. and Page, R. A. 2013. When to approach novel prey cues? Social learning strategies in frog-eating bats. Proc. R. Soc. B 280: 20132330.

9. Rafacz M, Templeton JJ. 2003 Environmental unpredictability and the value of social information for foraging starlings. Ethol. 109, 951-960.

10. van Bergen Y, Coolen I, Laland KN. 2004 Nine-spined sticklebacks exploit the most reliable source when public and private information conflict. Proc. R. Soc. B 271, 957-62.

11. Laland KN. 2004 Social learning strategies. Anim. Learn. Behav. 32, 4-14.

12. Webster MM, Ward AJ. 2011 Personality and social context. Biol. Rev. 86, 759-773. nonhuman primates. Adv. Stud. Behav. 34, 265-295.

382 attitudes: measurement, determinants, and behavioral consequences. J. Europ. Econ. Assoc. 9, 522-550. 
384 15. Reale D, Reader SM, Sol D, McDougall PT, Dingemanse NJ. 2007 Integrating animal 385 temperament within ecology and evolution. Biol. Rev. 82, 291-318.

386 16. Carter AJ, Marshall HH, Heinsohn R, Cowlishaw G. 2013 Personality predicts decision 387 making only when information is unreliable. Anim. Behav. 86, 633-639.

388 17. Harcourt JL, Ang TZ, Sweetman G, Johnstone RA, Manica A. 2009 Social feedback and the 389 emergence of leaders and followers. Curr. Biol. 19, 248-252.

390 18. Kurvers RH, van Oors K, Nolet BA, Jonker RM, van Wieren SE, Prins HH, Ydenberg RC.

3912010 Personality predicts the use of social information. Ecol. Lett. 13, 829-837.

392 19. Kurvers RH, Adamczyk VM, van Wieren SE, Prins HH. 2011 The effect of boldness on

393 decision-making in barnacle geese is group-size-dependent. Proc. R. Soc. B 278, 2018-

2024.

395 20. Byrnes JP, Miller DC, Schafer WD. 1999 Gender differences in risk-taking: a meta-analysis.

396 Psychol. Bull. 125, 367-383.

397 21. Charness G, Gneezy U. 2012 Strong evidence for gender differences in risk taking. J. Econ.

398 Behav. Org. 83, 50-58.

399 22. Cross CP, Cyrenne D, Brown GR. 2013 Sex differences in sensation-seeking: a meta-

$400 \quad$ analysis. Sci. Rep. 3, 2486.

401

23. Nelson JA. 2015 Are women really more risk-averse than men? A re-analysis of the literature 402 using expanded methods. J. Econ. Surv. 29, 566-585.

403 24. Harris CR, Jenkings M, Glaser D. 2006 Gender differences in risk assessment: why do 404 women take fewer risks than men? Judgm. Decis. Mak. 1, 48-63.

405 25. Weber EU, Blais A-R, Betz NE. 2002 A domain-specific risk-attitude scale: measuring risk 406 perceptions and risk behaviors. J. Behav. Dec. Making 15, 263-290.

407 26. Cross CP, Copping LT, Campbell A. 2011 Sex differences in impulsivity: a meta-analysis.

$408 \quad$ Psych. Bull. 137, 97-130.

409 27. Campbell A, Muncer S. 2009 Can 'risky' impulsivity explain sex differences in aggression?

$410 \quad$ Pers. Indiv. Diff. 47, 402-406. 
411 28. McElreath R. 2016 Statistical rethinking: A Bayesian course with examples in R and Stan 412 (Vol. 122). CRC Press.

413 29. Cross CP. 2010 Sex differences in same-sex direct aggression and sociosexuality: the role 414 of risky impulsivity. Evol. Psychol. 8, 779-792.

415 30. Bond R, Smith PB. 1996 Culture and conformity: a meta- analysis of studies using Asch's $416 \quad$ (1952b, 1956) line judgment task. Psych. Bull. 119, 111-137.

417 31. Morgan TJ, Rendell LE, Ehn M, Hoppitt W, Laland KN. 2012 The evolutionary basis of 418 human social learning. Proc. R. Soc. B 279, 653-662.

419 32. Cross CP, Brown GR, Morgan TJ, Laland KN. In press. Sex differences in confidence $420 \quad$ influence patterns of conformity. Brit. J. Psychol.

421 33. Gneezy, U., Niederle, M., \& Rustichini, A. (2003). Performance in competitive environments:

422 Gender differences. The Quarterly Journal of Economics, 118(3), 1049-1074.

423 34. Mesoudi A. 2011 An experimental comparison of human social learning strategies: payoff-

424 biased social learning is adaptive but underused. Evol. Hum. Behav. 32, 334-242.

425 35. Enquist M, Eriksson K, Ghirlanda S. 2007 Critical social learning: a solution to Rogers's 426 paradox of nonadaptive culture. Am. Anthropol. 109, 727-734.

427 36. Mishra S, Son Hing LS, Lalumière ML. 2015 Inequality and risk-taking. Evol. Psychol. 13, $428 \quad 1474704915596295$.

429 37. Atkisson C, O’Brien MJ, Mesoudi A. 2012 Adult learners in a novel environment use 430 prestige-biased social learning. Evol. Psychol. 10, 519-537.

431 38. Toelch U, Bruce MJ, Meeus MT, Reader SM. 2011 Social performance cues induce 432 behavioral flexibility in humans. Front. Psychol. 2, 160.

433 39. Jansen, B.R., van Duijvenvoorde, A.C. and Huizenga, H.M., 2014. Developmental and 434 gender related differences in response switches after nonrepresentative negative feedback. 435 Dev. Psychol., 50, 237-246. 
436 40. Mesoudi A, Chang L, Dall SR, Thornton A. 2016 The evolution of individual and cultural 437 variation in social learning. Trends Ecol. Evol. 31, 215-225.

438 41. Kahneman, D., \& Tversky, A. (2013). Prospect theory: An analysis of decision under risk. In Handbook 439 of the Fundamentals of Financial Decision Making: Part I (pp. 99-127). 


\section{Table $\mathbf{1}$ (on next page)}

Candidate models and their WAIC weights

List of candidate models that were included in the asocial/social information analysis, the a priori hypotheses and the included parameters, with model values (WAIC \pm SE) and weights (Akaike weight). Bold type indicates the best fitting model 
1

2 Table 1 List of candidate models that were included in the asocial/social information analysis,

3 the a priori hypotheses and the included parameters, with model values (WAIC \pm SE) and

4 weights (Akaike weight). Bold type indicates the best fitting model.

5

\begin{tabular}{|c|c|c|c|c|}
\hline Model & Hypothesis & Parameters included & $\begin{array}{l}\text { WAIC } \\
( \pm S E)\end{array}$ & $\begin{array}{l}\text { Akaike } \\
\text { weight }\end{array}$ \\
\hline 1 & Null & Intercept & $\begin{array}{l}360.3 \\
(5.10)\end{array}$ & 0.00 \\
\hline 2 & Full & $\begin{array}{l}\text { Intercept + sex + AR + SR + } \\
\text { sex*AR + sex*SR + rank + } \\
\text { personality }\end{array}$ & $\begin{array}{l}357.1 \\
(10.36)\end{array}$ & 0.01 \\
\hline 3 & $\begin{array}{l}\text { Sex and condition interactions } \\
\text { predict choice }\end{array}$ & Intercept + sex $^{*} A R+$ sex $^{*} S R$ & $\begin{array}{l}351.1 \\
(9.11)\end{array}$ & 0.25 \\
\hline 4 & $\begin{array}{l}\text { Sex, and sex and condition } \\
\text { interactions, predict choice }\end{array}$ & $\begin{array}{l}\text { Intercept + sex + sex*AR + } \\
\text { sex*SR }\end{array}$ & $\begin{array}{l}349.6 \\
(9.77)\end{array}$ & 0.52 \\
\hline 5 & $\begin{array}{l}\text { Sex and condition predict } \\
\text { choice }\end{array}$ & Intercept + sex + AR + SR & $\begin{array}{l}354 \\
(8.92)\end{array}$ & 0.06 \\
\hline 6 & Only condition predicts choice & Intercept + AR + SR & $\begin{array}{l}352 \\
(8.77)\end{array}$ & 0.16 \\
\hline 7 & Only sex predicts choice & Intercept + sex & $\begin{array}{l}362.3 \\
(5.32)\end{array}$ & 0.00 \\
\hline
\end{tabular}

6 
Figure 1

Screenshot of Phase1

Example screenshot from the online experiment showing shipbuilding in Phase 1.

\section{You are on Phase 1 of ROUND 1}

Perishable foodstuffs
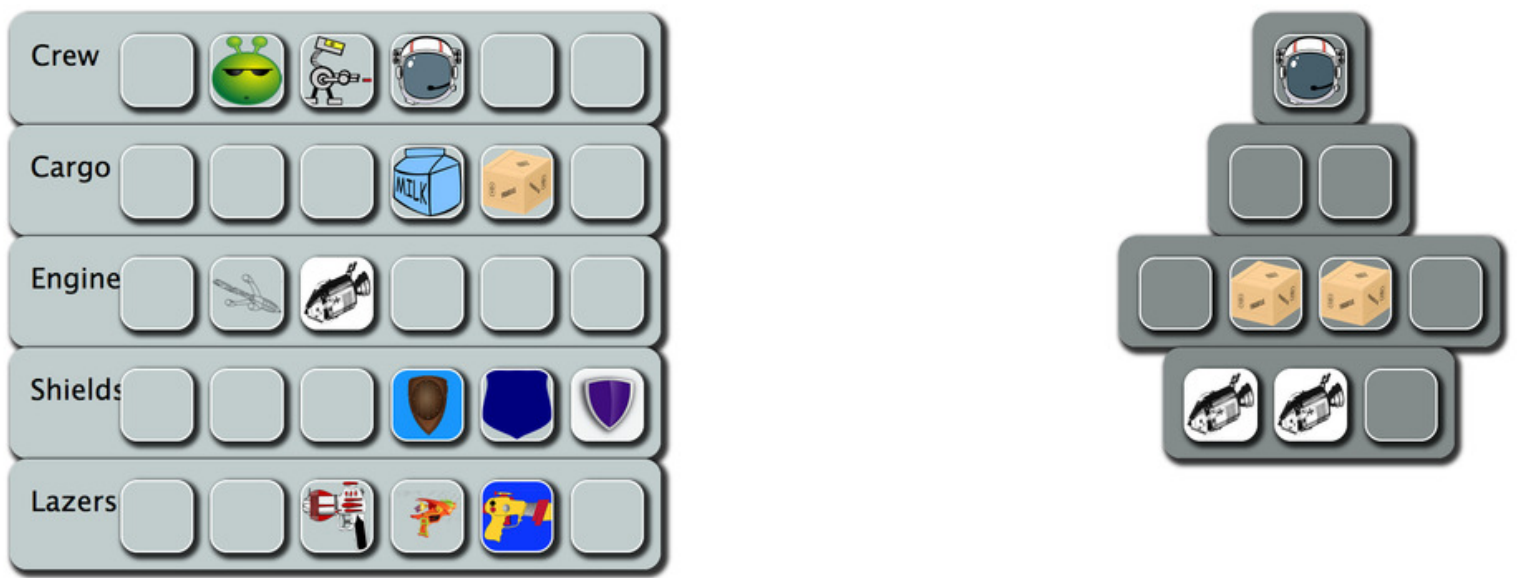

REMEMBER:
$\begin{aligned} & \text { You have to use at least one engine and one crew member - and you only } \\ & \text { have TWO MINUTES! }\end{aligned}$

Time Remaininq: 01:11 
Figure 2

Screenshot of Phase1.

Example screenshot of the generated score presented to participants at the end of Phase 1.

\section{You have completed Phase 1}

Well done! Your ship's score is 25,000 . Here is a list of the high scores:

\begin{tabular}{|r|r|}
\hline Name & \multicolumn{1}{|c|}{ Score } \\
\hline Rank 1 & 25,000 \\
\hline Rank 2 & 21,050 \\
\hline Rank 3 & 16,850 \\
\hline Rank 4 & 10,500 \\
\hline Rank 5 & 8,780 \\
\hline
\end{tabular}

Click continue to find out how you can improve your score!

Continue 


\section{Figure 3}

Screenshot of the choice participants saw

Screenshot of the two choices participants saw in the two conditions. The top two choices were displayed in the Risky Social condition, the bottom two were displayed in the Risky Asocial condition. Which option was displayed on the right or left was randomised in both conditions. 


\section{Please choose your next step:}

See other players' ships

You will be shown three spaceships built by previous players. Some of the spaceships might be well-built, some of them might be poorly built and unable to fly. The ships may have a much worse or much better score than your current ship's score. You will get to choose three items from one of the ships to copy. Depending on which you choose, your score could go up or down.

Select

\section{Spaceship Scrapheap}

You will be shown the "spaceship scrapheap" which contains a large collection of items that you have not already seen. All of these items will help your ship to fly, and some of them can slightly increase your ship's score. You will get to choose three items from the scrapheap to use for your next ship, and will be guaranteed at least the same score as your current ship.

\section{Select}

\section{Please choose your next step:}

\section{Spaceship Scrapheap}

You will be shown the "spaceship scrapheap" which contains a large collection of items that you have not already seen. Some of these items may be broken and useless, but some may greatly increase your ship's score. You will get to choose three items from the scrapheap to use for your next ship, but depending on which you choose, your score could go up or down.

\section{Select}

See other players' ships

You will be shown three spaceships built by previous players. All of the spaceships are well-built and are able to fly. The ships will have the same score as your ship, or slightly higher. You will get to choose three items from one of the ships to copy, and will be guaranteed at least the same score as your current ship.

\section{Select}




\section{Figure 4}

\section{Model predictions}

Model predictions of the mean proportion of individuals that chose social information plotted according to condition and sex. Predictions were averaged across all models and weighted according to WAIC weight. Error bars show $89 \%$ Cls. Raw means are also plotted, represented by a cross symbol

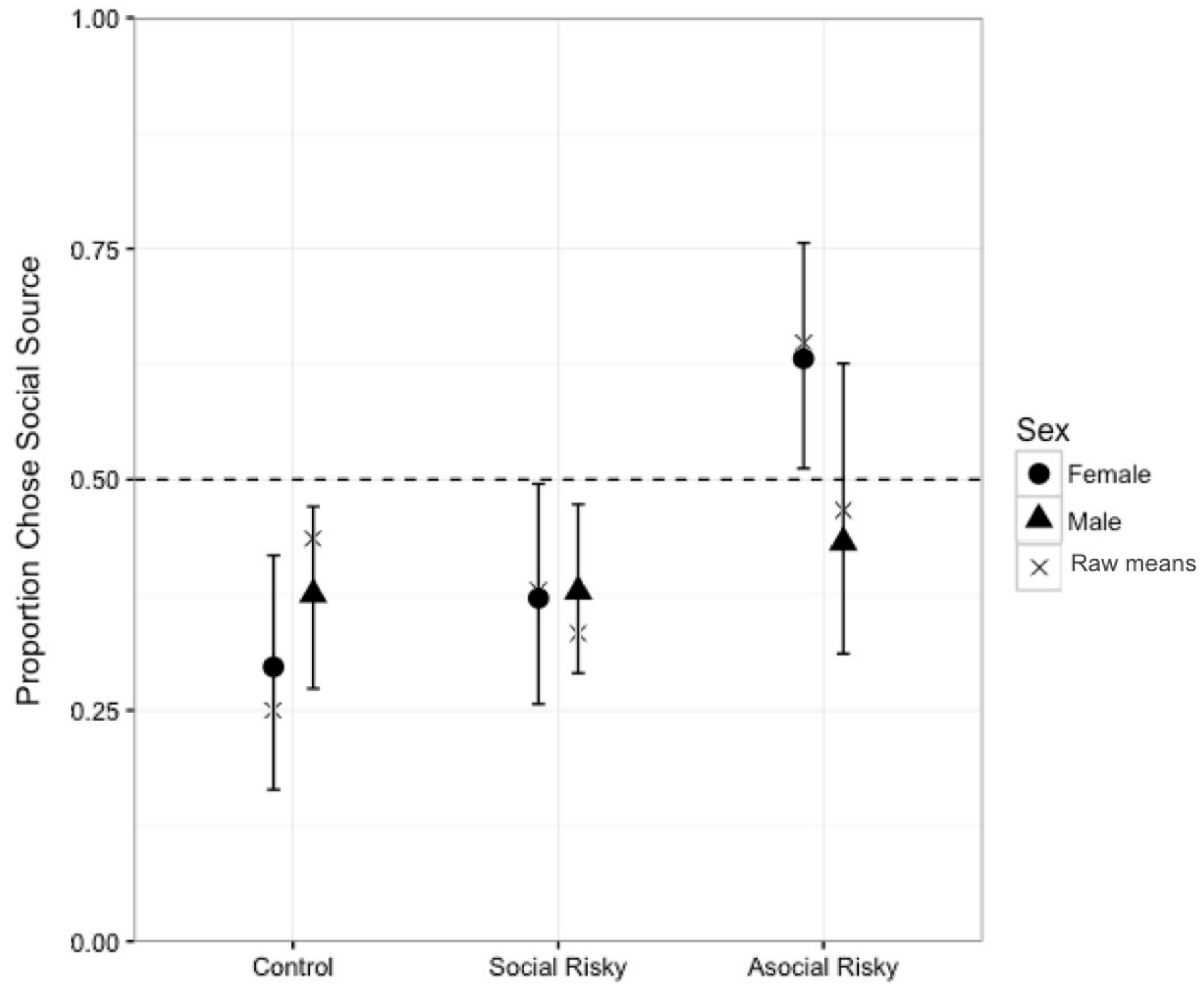




\section{Figure 5}

Plot of parameter estimates of best fitting model (lowest WAIC)

Plot displaying parameter estimates for the probability of choosing the social option, taken from the model with the lowest WAIC value and plotted with $89 \% \mathrm{Cls}$. A positive estimate indicates a greater likelihood of choosing social, rather than asocial, information. Where the $89 \%$ Cls of parameter estimates include zero, there is no clear evidence of an effect of that parameter on the likelihood of choosing the social or asocial option. The intercept (baseline) represents males in the control condition

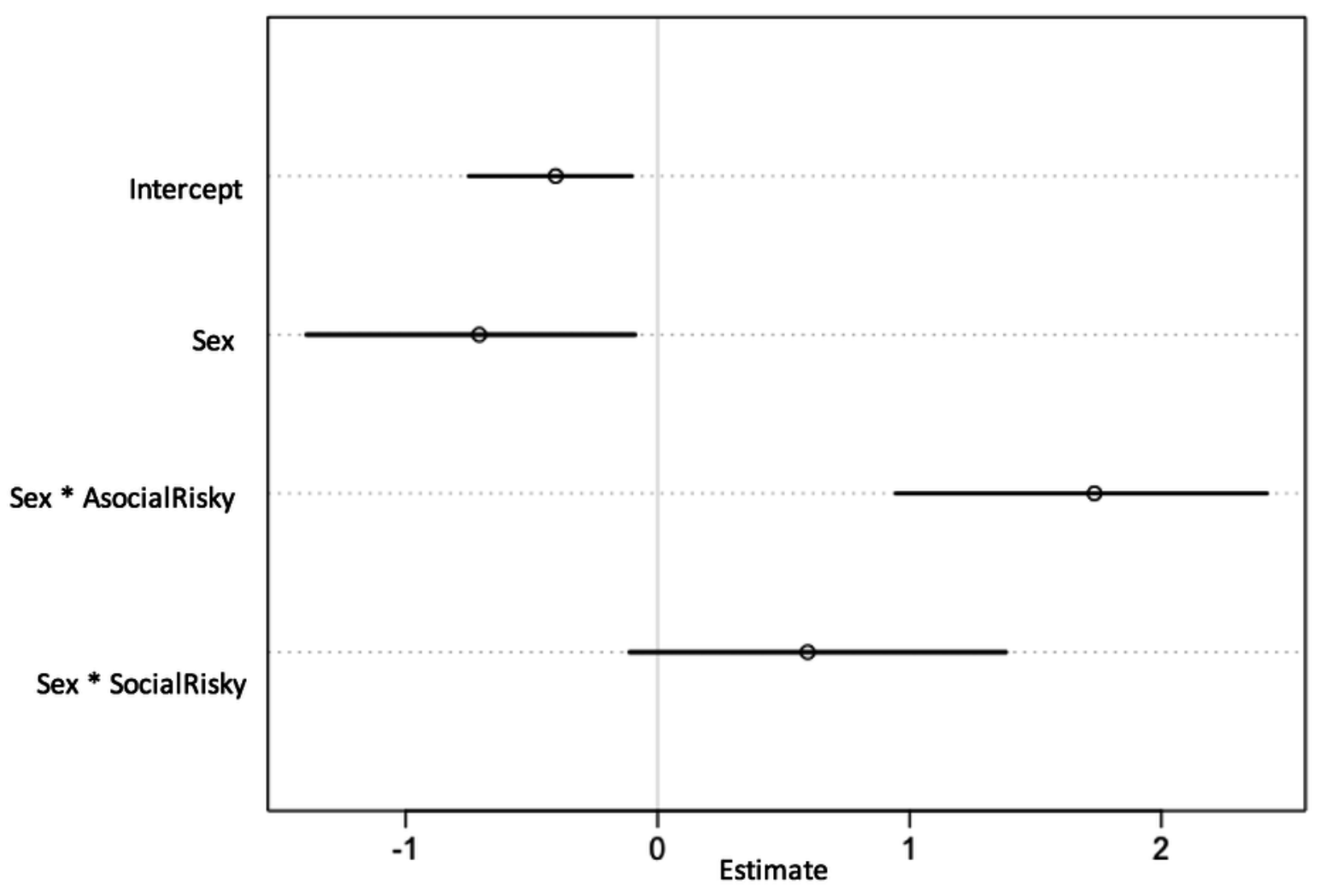




\section{Figure 6}

Plot of parameter estimates from Full Model.

Plot displaying parameter estimates for the probability of choosing the social option, taken from the full model and plotted with $89 \%$ Cls.

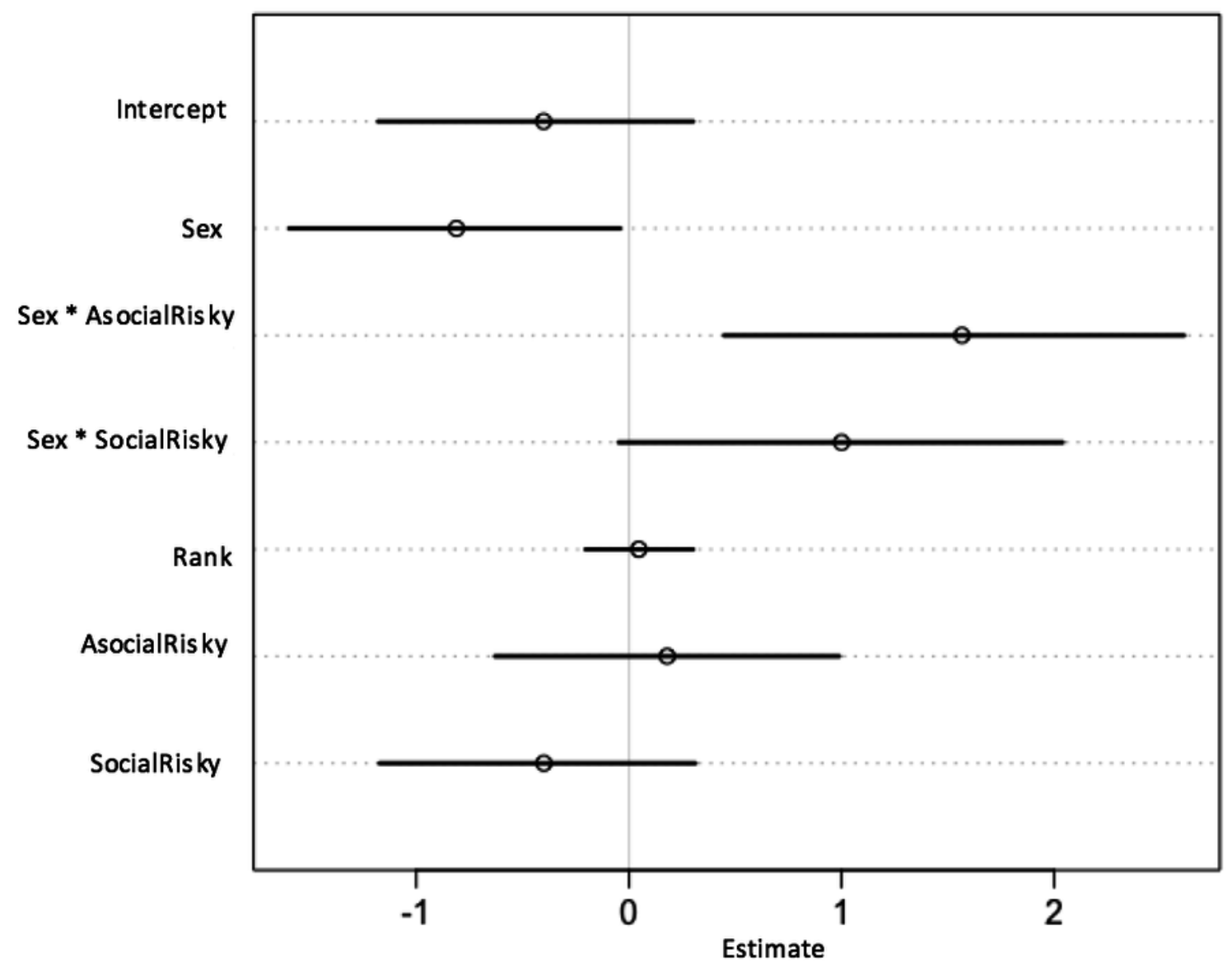


Figure 7

Plot of parameter estimates from the second model.

Plot displaying parameter estimates for the likelihood of choosing the risky option, plotted with $89 \%$ Cls. A positive estimate indicates a greater likelihood of choosing the risky, rather than the safe, option. Where estimates include zero, there is no clear evidence of that parameter affecting the likelihood of choosing the risky or safe option




Figure 8

Density plot of sex difference in risk-taking measure

Density plot showing men and women's risky impulsivity scores

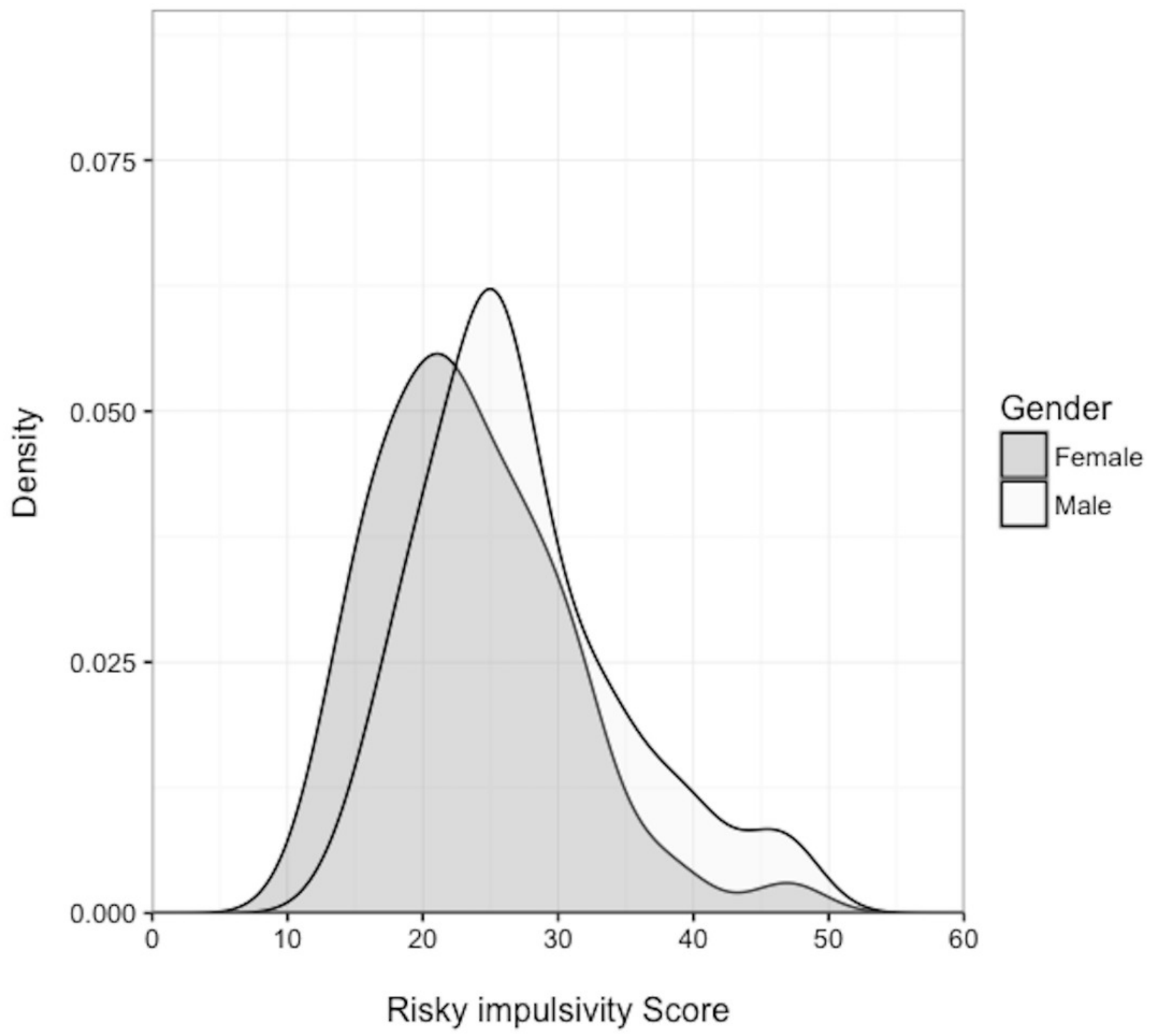

